\title{
Noise Reduction Using an Adaptive Microphone Array in a Car -A Speech Recognition Evaluation
}

\author{
S. Noniebo S. Nordholm \\ Department of Signal Processing, University of Karlskrona/Ronneb y, Ronneby \\ B. Bengtsson I. Claesson \\ Dept. of Telecom. Theory, University of Lund
}

\begin{abstract}
This paper describes an evaluation of an adaptive microphone array with respect to speech recognition performance in a car. The microphone array is compared to two conventional microphones of different types. The speech recognition device is aimed to be a part of a man/machine-interface between the driver and car information services.
\end{abstract}

\section{Introduction,}

In order to enhance traffic safety and keep the drivers' attention to the traffic, it is suitable to use speech synthesis to present information and speech recognition to control the flow of information. Due to the noisy situation in a car it is necessary to employ some noise reducing device in order to make the speech recognition function satisfactorily.

This paper ${ }^{\perp}$ presents an evaluation where recordings from a large speech material have been used in a car in typical traffic situations. Utterances have been recorded with two different conventional microphones and a microphone array. The array recordings have been processed in a signal processing system DSP900, where a spatial filtering technique[1] in conjunction with adaptive filters has been used. The material has been evaluated with respect to speech recognition performance.

\section{Spatial Filters for the GSC}

A Linear Array Broadband Generalized Sidelobe Canceller[2] (GSC) consists of an array with an upper beamformer $G$ providing the "desired response" to the adaptive algorithm and lower beamformers $B_{i}$ which provide the inputs to the adaptive filters $\mathbf{H}_{\text {\# }}$ see fig. 1 . The purpose of the lower beamformers is to protect the target signal from being cancelled by the adaptive filters and 1-D spatial filters have been used for this purpose(1).

${ }^{1}$ This work is ponsored by NUTEK and supported by the Swedish RTI-program (Road Transport Informatic) which is baced on two European projects, Prometheus and Drive. The purpose of these projects is to develop chs application of modern information technoiogy for transport. Improved trafic information should give shorter transport times, les fuel consumption and fewer accidents.

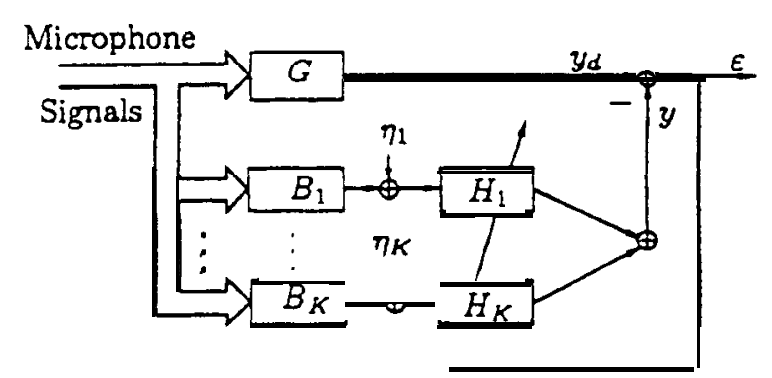

Figure 1: Adaptive beamformer structure

Zero weigths in the adaptive filters when no signals are incident on the array are often wanted [3]. This is easily obtained if a leaky algorithm is used to update the adap tive filters, provided the "leaky" noise $\boldsymbol{\eta}_{\mathbf{i}}$ dominates over the sensor noise. Furthermore, a dominating "leaky" noise implies simple Wiener solutions and straightforward design criteria for the spatial filters $\boldsymbol{B}_{\mathbf{i}}$. In figure 2 the performance of the adaptive array is illustrated. Utterances are made as five words-pause-five words and the output power leve1 with and without noise cancelling is plotted. A typical 10-15 dB improvement is obtained.

\section{The Speech Recognition}

The speech-recognition device is a modified version of the Infovox RA-201, marketed by Infovox AB. This is a speaker-adaptive system using template matching of cepstral coefficients[4]. The input is preemphasized by +6 dB per octave between 200 and $5000 \mathrm{~Hz}$, bandlimited for anti-aliasing and sampled with a rate of $10 \mathrm{kHz}$. The digitized signal is then processed by a NEC 7720 signal processor used as a $\mathbf{1 6}$ channel Bark-scale filter bank. Seven of the eight lowest cepstral coefficients are calculated from the amplitudes in the 16 Bark-scale bands with a rate of $40 \mathrm{~Hz}$. The first coefficient contains the overall energy and is not used, and therefore the seven cepstral mefficients describes the shape of the spectrum which is not sensitive to signal amplitude. After endpoint detection, the words arc linearly normalized to 32 samples which implies a nominal length of $32 \cdot 1 / 40$ s. During remgnition, the unknown utterance is nonlinearly time-normalized for optimal alignment to each 


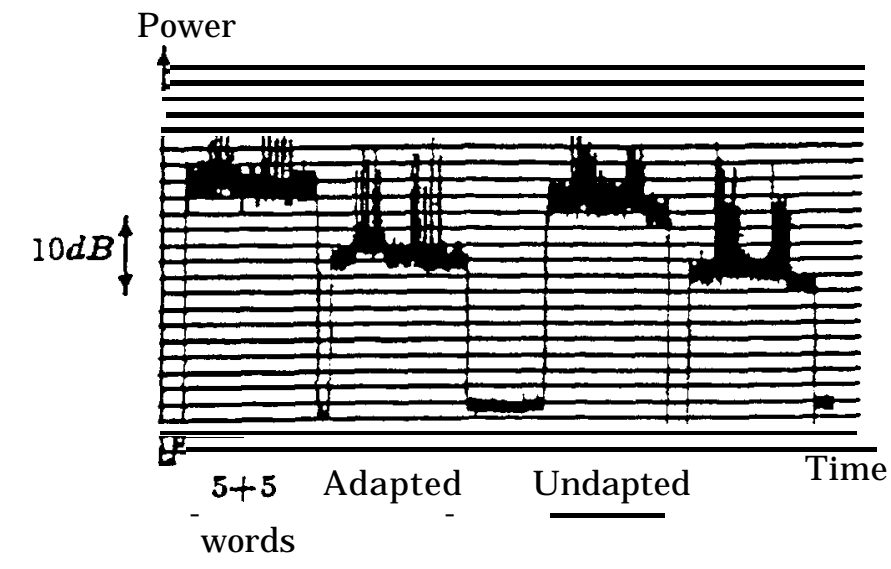

Figure 2: Illustration of SNR-improvement with adap tive array

vocabulary word.

A noise compensation technique hss been used, where the recognition system is adapted to environmental noise. The noise is measured just before and/or after the sampled word and is "added" [4] to the reference templates which am recorded in a silent environment. This procedure compensates for the fact that training and recognition have occurred under different environmental conditions.

\section{Results}

The results presented here are based on six speakers, three women and three men reading fifty-three different utterances repeated ten times each. However, the last two females spoke considerably lower than the other four speakers, making it almost impossible to detect words for the more distant microphones. The material is evaluated with respect to speech recognition, see fig. 3 and fig. 4.

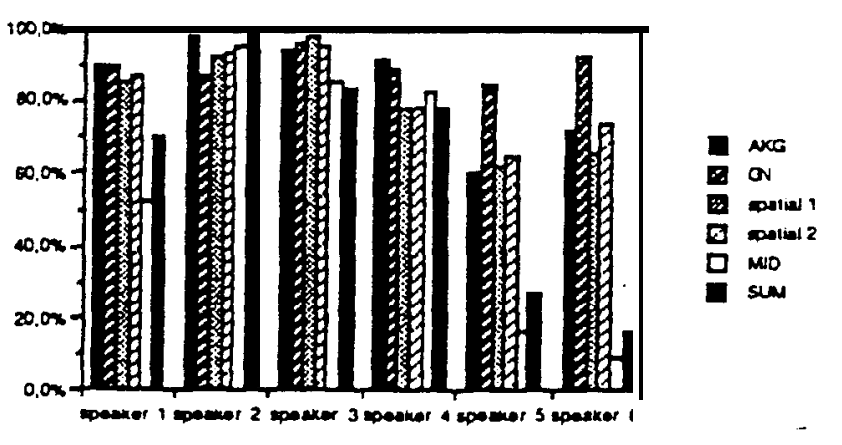

Figure 3: Gorrectly recognized utterances in percent
The speech material is recorded in a studio and played with a loudspeaker in a cardriving $90 \mathrm{~km} / \mathrm{h}$. Simultaneous recordings are roade in the car with a bigh quality condenser microphone (AKG), a goose-neck microphone (GN) especially designed for mobile telephony and an eight-element array of medium quality condenser microphones. The array recordings are processed using two particuiar 1-D spatial filter designs (spatial 1 and spatial 2). One of the array microphones (MID) and the sum of the eight (SUM) are included in the test. The array and AKG microphones are placed at approximately the same distance $(40-50 \mathrm{~cm})$ in front of the speaker, while the goose-neck microphone is placed at a distance less than $10 \mathrm{~cm}$.

The results should be interpreted with care since there is a large variation among speakers. In fig. 3, GN is the most robust microphone, while MID, which is the simplest microphone, and AKG, which is the most expensive, perform poorly when the speaker signal is weak (speaker 5 and 6). In fig. 3, spatial 1 and spatial 2 perform significantly better than MID and SUM as expected. The high quality AKG shows the highest detection rate for speaker 1 through 4 (as expected). The GN performs approximately as spatial 1 and spatial 2 despite the fact that it is mounted very close to the speaker.

Further, fig. 4 shows that the "false alarm rate" is significant for the GN microphone due to the commercial mounting. The other microphones are mounted acoustically correctly.

\section{Possible improvements and current efforts}

The speech recognition algorithm does not take into account the loss of lower frequencies caused by the adap tive array. The anay works as an active microphone and

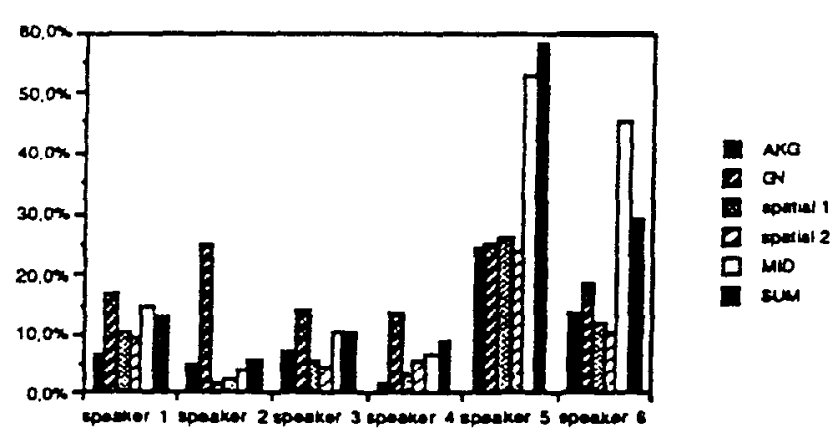

Figure 4: Falsely detected in percent (false alarms) 
the frequency response depends on the noise situation, i.e the microphone will have different frequency characteristics at training and at recognition, the difference can be as much ss $20 \mathrm{~dB}$ for some frequencies. However, the speech recognition device is based on power measurements in different frequency bands and a frequency distortion might therefore be devastating. If the algorithms could compensate for the different frequency characteristics at training and recognition, the adaptive array would be better exploited. In our future work this will be taken into account. Further a possible improvement is to use microphones of better quality in the array (at least as good as the goose-neck microphone).

A cumbersome part of the spatial filter realization is the calibration of the microphones and analog channels at the inputs. A self calibrating realization has therefore been developed. Apart from being very robust against channel mismatch preliminary results indicate a further $5 \mathrm{~dB}$ improvement of the signal-tc-noise ratio, yielding even better recognition performance.

\section{Summary and Conclusions}

A broadband adaptive microphone array has been tested and compared to conventional microphones with respect to speech recognition in a car. The measurements show that the array gives a higher signal to noise ratio than the other microphones, and is as good as a goose-neck microphone for speech recognition.

\section{References}

1. 1. Claesson and S. Nordholm, "A Spatial Filtering Ap proach to Robust Adaptive Beamforming", IEEE Transactions on Antennes \& Propagation, September 1992.

2. B.D. van Veen, K.M. Buckley, "Beamforming: A Versatile Approach to Spatial Filtering", IEEE ASSP Magazine, April 1988.

3. L.J. Griffiths, K.M. Buckley, “Quiescent Pattem Control in Linearly Constrained Adaptive Arrays", IEEE ASSP Transactions, July 1987.

4. M. Blomberg, K. Elenius, "Optimizing some parameters of a word recognizer used in car noise", Speech Trensmisszon Laboratory Quarteriy Progress and Statw Report, $4 / 1990, \mathrm{KTH}$ 